\title{
The effect of xenon-augmented sevoflurane anesthesia on intraoperative hemodynamics and early postoperative neurocognitive function in children undergoing cardiac catheterization: A randomized controlled pilot trial
}

\author{
Sarah Devroe $^{1}$ (D) | Jurgen Lemiere ${ }^{2,3}$ | Laura Van Hese ${ }^{4}$ | Marc Gewillig ${ }^{4,5}$ | \\ Derize Boshoff $^{5}$ | Koen Poesen ${ }^{6,7}$ | Marc Van de Velde ${ }^{1,4}$ Steffen Rex ${ }^{1,4}$
}

\footnotetext{
${ }^{1}$ Department of Anesthesiology, University Hospitals Leuven, Leuven, Belgium

${ }^{2}$ Department of Child and Adolescent Psychiatry, University Hospitals Leuven, Leuven, Belgium

${ }^{3}$ Department of Paediatric HaematoOncology, University Hospitals Leuven, Leuven, Belgium

${ }^{4}$ Department of Cardiovascular Sciences, KU Leuven, Leuven, Belgium

${ }^{5}$ Department of Pediatric and Congenital Cardiology, University Hospitals Leuven, Leuven, Belgium

${ }^{6}$ Department of Laboratory Medicine, University Hospitals Leuven, Leuven, Belgium

${ }^{7}$ Department of Neurosciences, KU Leuven, Leuven, Belgium
}

Correspondence: Dr Steffen Rex, MD, PhD, Department of Anesthesiology, University Hospitals Leuven, Herestraat 49, 3000 Leuven, Belgium (steffen.rex@uzleuven.be).

Funding information

The study was funded by departmental resources.

Section Editor: Laszlo Vutskits

\section{Summary}

Background: In adults, xenon has only minimal hemodynamic side effects when compared with other anesthetics. Moreover, in preclinical experiments, xenon has been demonstrated to possess cardio- and neuroprotective properties. Altogether, the favorable hemodynamic profile combined with its potential for organ-protection could render xenon an attractive option for anesthesia in children with cardiovascular compromise.

Aims: The aim of this study was to explore safety and feasibility of sevoflurane-augmented xenon anesthesia in school-aged children and to assess early postoperative neurocognitive effects of xenon-sevoflurane and sevoflurane anesthesia when compared to a control group that did not have anesthesia.

Methods: Forty children aged 4-12 years, suffering from congenital heart disease, undergoing diagnostic or interventional cardiac catheterization were randomized to either xenon-augmented sevoflurane anesthesia or sevoflurane alone. Safety was assessed by the incidence of intraprocedural hemodynamic instability and feasibility by anesthetic depth and respiratory profile. In addition, neurocognitive performance was assessed preoperatively, 2 hours after discharge from PACU and at 24 hours after anesthesia using the Amsterdam Neuropsychological Tasks system. A healthy control group of 22 age- and gender-matched children not exposed to anesthesia underwent an identical neurocognitive test battery, at comparable time intervals.

Results: Overall hemodynamics did not differ between groups. Xenon-sevoflurane anesthesia resulted in decreased intraoperative ephedrine requirements (median [IQR]) $(0.00 \mathrm{mg} / \mathrm{kg}$ [0.00-0.00] vs $0.00 \mathrm{mg} / \mathrm{kg}$ [0.00-0.01], $P=0.047)$. Only neurocognitive tests in the domain of alertness were significantly impaired 2 hours postoperatively in both anesthesia groups in comparison with the control group (alertness variability: $P=0.02$, odds ratio 5.8 ), but recovered at 24 hours. For working memory, inhibition, cognitive flexibility, and motor coordination 
tasks, no significant interaction effects of anesthesia were found in the early postoperative period.

Conclusion: In this pilot trial, xenon-augmented sevoflurane anesthesia in schoolaged children was feasible; and associated with decreased ephedrine requirements. All children exposed to anesthesia showed impaired neurocognitive performance in the immediate postoperative period when compared to control children; however, without significant differences between both treatment groups.

KEYWORDS

anesthetics, child, hemodynamics, inhalation, postoperative neurocognitive outcome, recovery, school, sevoflurane, xenon

\section{1 | INTRODUCTION}

In adults, the noble gas xenon has only minimal hemodynamic side effects when compared with other anesthetics. ${ }^{1-3}$ Moreover, in preclinical experiments, even subanesthetic doses of xenon have been demonstrated to possess cardio- and neuroprotective properties ${ }^{4,5}$ and to protect from anesthetic neurotoxicity. ${ }^{6}$ Altogether, the favorable hemodynamic profile combined with its potential for organ-protection could render xenon an attractive option for anesthesia in children suffering from congenital heart disease (CHD). Unfortunately, data on the use of xenon in pediatric anesthesia are scarce.

Owing to its low potency, ${ }^{7}$ xenon cannot be used as a monoanesthetic in children and needs to be added to another anesthetic to allow for an adequate depth of anesthesia. In line with this, our group could recently demonstrate that xenon-augmented sevoflurane anesthesia in children aged 0-4 years undergoing diagnostic or interventional cardiac catheterization was safe, feasible, and facilitated hemodynamic management. ${ }^{8}$

To the best of our knowledge, there are no data available for the effect of xenon anesthesia on children between 4 and 12 years of age. Studying this age group also allows investigating postoperative cognitive function, using validated age-adjusted neuropsychological tests.

In this randomized controlled trial, we studied the safety and the feasibility of xenon-augmented sevoflurane anesthesia in school-aged children. Moreover, we tested cognitive performance early after xenon-augmented- and mono-sevoflurane anesthesia in comparison to a healthy control group receiving no anesthesia.

\section{MATERIALS AND METHODS}

\section{1 | Study design and study subjects}

The present study is a phase II mono-center, prospective, single-blind randomized controlled pilot trial, performed according to the principles of good clinical practice and the International Declaration of Helsinki. The study protocol was approved by the local ethics committee (SR56902, Commissie Medische Ethiek, Universitaire

\section{What is already known}

- In adults, xenon has only minimal hemodynamic side effects when compared to other anesthetics.

- Xenon conveys neuroprotective properties in experimental models mimicking neonatal neuronal injury, and protects against developmental neurotoxicity induced by other anesthetics.

- Experience with xenon anesthesia in children is scarce.

\section{What this article adds}

- Combining xenon with sevoflurane in children between 4 and 12 years of age is safe and feasible.

- In children between 4 and 12 years of age with congenital heart disease undergoing cardiac catheterization, xenon-augmented sevoflurane anesthesia does not improve hemodynamic stability but reduces ephedrine requirements.

- sevoflurane anesthesia results in short-lasting neurocognitive deficits, which cannot be ameliorated by xenon.

Ziekenhuizen Leuven, September 17th, 2014) and by the Federal Agency for Medicines and Health Products, Brussels, Belgium (AFMPS/R\&D/CED/mm830076, September 5th, 2014). The study is registered at the European Medicines Agency (EudraCT: 2014 002510-23) and reported according to the CONSORT statement.

The study protocol has been described in detail. ${ }^{9}$ After obtaining written informed parental consent and an age-adjusted children's assent, 62 (40 anesthesia-exposed and 22 control) children were enrolled in this trial.

Children were eligible if they were between 4 and 12 years of age. The intervention groups had to be scheduled for a diagnostic or interventional cardiac catheterization. Exclusion criteria were: an expected intraprocedural oxygen requirement above $40 \%$, necessity to undergo a high-risk interventional procedure (as defined by the pediatric interventional cardiologist), psychomotoric retardation (as 
determined by the nonachievement of age-specific developmental milestones), and a lack of written parental informed consent or patients' assent. The children were randomized using a softwaregenerated permuted block randomization sequence (Sealed EnvelopeTM, London, UK) to receive anesthesia with either sevoflurane alone (sevoflurane group) or xenon as an adjuvant to sevoflurane (xenon group). They were allocated based on a closed envelope method [1:1 ratio, stratified by age (stratum I: age 4-7 years; stratum II: 8-12 years)]. Two types of investigators conducted the trial. Investigator I accomplished the enrollment on the day before the intervention and performed all postoperative visits. He was, similar to the patient and his parents, blinded to treatment allocation. Investigator II conducted the GA and was necessarily unblinded to the treatment due to the treatment conditions (management of either one or two inhalational anesthetics).

Twenty-two healthy children matched for age and gender were included as control group for the neurocognitive assessments.

\section{2 | Anesthesia, intervention, and postinterventional follow-up}

Preoperative anxiolytic drugs were omitted (to avoid interference with recovery characteristics), but parents were encouraged to accompany their children at the induction of anesthesia. Noninvasive cardiorespiratory and bispectral index (BIS) (pediatric sensor, Medtronic-Covidien, Minneapolis, US) monitoring were established according to institutional standards. For the induction of anesthesia, fentanyl $(2 \mu \mathrm{g} / \mathrm{kg}$ ), propofol (3 $\mathrm{mg} / \mathrm{kg}$ ), and rocuronium $(0.3 \mathrm{mg} / \mathrm{kg}$ ) were administered intravenously (IV). All children were endotracheally intubated. Dexamethasone $(0.15 \mathrm{mg} / \mathrm{kg})$ was given to prevent postoperative nausea and vomiting (PONV). If an IV line was not available, anesthesia was induced by sevoflurane inhalation. Then, the randomization envelope was opened, and GA was maintained with either $50 \%-65 \%$ xenon (LENOX ${ }^{\mathrm{TM}}$, AirLiquide Santé International, Paris, France) in oxygen $\left(\mathrm{FiO}_{2}=0.25-0.4\right)$ as an adjuvant to sevoflurane (xenon group), or sevoflurane (Sevorane, AbbVie, Wavre, Belgium) alone $\left(\mathrm{FiO}_{2}=0.25-0.4\right)$ (sevoflurane group), using a closedcircuit respirator (FelixDual ${ }^{\mathrm{TM}}$, AirLiquide Medical Systems, Paris, France). To achieve a comparable depth of anesthesia in both groups, sevoflurane concentrations were titrated to achieve BIS values of 40-60 and physiological signs suggestive for an appropriate depth of anesthesia. Investigator II decided on the administration of additional opioids or muscle relaxants. The treatment of hemodynamic instability was standardized but left at the discretion of investigator II. Isolated blood pressure declines $>20 \%$ from baseline were treated with a bolus of phenylephrine $(2-3 \mu \mathrm{g} / \mathrm{kg})$ and/or a bolus of fluid (crystalloid $10 \mathrm{~mL} / \mathrm{kg}$ ), isolated bradycardia with a bolus of atropine $(10-20 \mu \mathrm{g} / \mathrm{kg})$, and the combination of bradycardia with hypotension with a bolus of ephedrine $(50-100 \mu \mathrm{g} / \mathrm{kg})$. Fluid management was performed according to the "4/2/1-rule" ( $\mathrm{mL} / \mathrm{kg} / \mathrm{h}$ ) using a balanced crystalloid solution. All children received paracetamol $(15 \mathrm{mg} / \mathrm{kg}$ ) for postoperative analgesia. After the end of the procedure, the children were extubated and transferred to the postanesthesia care unit (PACU). Cardiopulmonary and safety parameters were assessed at the PACU, 1 hour after discharge from the PACU and on the following morning.

\section{3 | Outcome}

The primary outcome of this clinical trial was to evaluate safety and feasibility of xenon anesthesia in children.

1. Safety was assessed by the incidence of intraprocedural hemodynamic adverse events (not caused by obvious interventional manipulation), defined by a $>20 \%$ change from baseline for $H R$ or MAP or by the need for fluid boluses, vasopressors, inotropes, or chronotropes.

2. Feasibility was assessed by:

i. Depth of anesthesia, assessed by BIS values and clinical signs (movements or sudden changes in HR or MAP).

ii. Intraoperative respiratory profile [measured by arterial oxygen saturation $\left(\mathrm{SpO}_{2}\right)$ and endtidal $\mathrm{CO}_{2}$ concentrations].

\subsection{Secondary endpoints}

\subsection{1 | Cognitive assessment}

Computerized tasks of the Amsterdam Neuropsychological Tasks (ANT) system ${ }^{10}$ were administered the day before the intervention, 2 and 24 hours postoperatively by investigators blinded to the treatment allocation. The selection of ANT tasks covers a variety of cognitive functions: alertness, divided attention, working memory, inhibitory control, cognitive flexibility, and motor coordination. Alertness is measured with the baseline tasks of the ANT. Reaction time (RT) and variability of RT are used as outcome measures for alertness. Divided attention is measured with the subtest memory search objects or letters. Both speed and accuracy measures are recorded. Working memory is assessed with the same tasks, but memory load is increased. The response organization objects task is used to measure inhibition and cognitive flexibility and speed and accuracy scores are used. Difference scores between tests of increasing demand are calculated to obtain pure measures of working memory, inhibition or cognitive flexibility. Finally, the subtest finger tapping is used to assess motor coordination. The number of correct taps on four conditions is recorded: (non)-dominant hand, bimanual alternating, and bimanual synchronous.

To check whether the three groups were comparable for general intelligence and psychosocial functioning the following baseline measures were performed: First, all patients performed a baseline intellectual testing with the short version of the Wechsler Preschool Primary Scale of Intelligence-III (WPPSI-III) for children $<6$ years, and the Wechsler Intelligence Scale of Children (WISC-III) for patients between 6 and 16 years. Second, two questionnaires were completed at the first visit by one of the parents. The Behavioral Rating Inventory of Executive Functions (BRIEF) assesses different executive functions of children, such as inhibition, cognitive flexibility, 
emotion regulation, taking initiative, working memory, planning and organizing, orderliness, and monitoring of behavior. The Child Behavior Checklist (CBCL) 1.5-5 and 6-18 years is a standardized questionnaire for evaluating psychosocial problems.

\subsubsection{Recovery parameters}

1. Time to extubation and to open eyes (measured from discontinuation of the investigational treatment).

2. Aldrete score, at $5,10,15,30,45$, and 60 minutes after extubation.

3. Recovery index: $\mathrm{RI}=\frac{1+\text { Aldrete score at T5 }}{(2 \text { time to extubation })+\text { time to open eyes }}{ }^{11}$

4. Readiness for discharge from the PACU (defined by the time to reach an Aldrete score of $\geq 9$ ).

5. Duration of PACU stay.

6. Incidence of emergence delirium (ED), assessed by the "Pediatric Anesthesia Emergence Delirium Scale" (PAED-scale) and the "4point Agitation Scale" (Watcha scale), every 5 minutes from the moment of extubation until discharge from the PACU. ED was diagnosed if patients had a score of $\geq 3$ on the Watcha scale or $\geq 12$ on the PAED-scale. ${ }^{12}$

7. Incidence of PONV. PON was assessed by a visual analog score (0-100) and POV by the number of emetic episodes.

\subsection{3 | Other}

1. Blood levels of serum protein $100 \beta$ (S100 $\beta$ ), IL-6, and IL-10.

2. Intraoperative consumption of xenon, sevoflurane, and fentanyl.

3. The incidence of (serious) adverse events [(S)AEs].

\section{5 | Statistical analysis}

The sample size was based on convenience because data on the hemodynamic and neurocognitive effects of xenon in children were not available in the literature when planning this trial. Based on various xenon trials of our group ${ }^{3,13}$ and other investigators ${ }^{14}$ in adults suffering from cardiac disease, a number of 40 children was expected to be sufficient to detect statistically significant differences in safety and efficacy parameters. A comparable sample size has also been used in several studies investigating postoperative cognitive functioning in children. ${ }^{15-17}$

Statistical analysis was performed using SPSS 24 software (SPSS Statistics version 24 for Windows, IBM, Armonk, New York, United states) according to the intention-to-treat principle. Data were tested for normality using the Shapiro-Wilk test. Continuous variables are reported using median and interquartile range (IQR) and were compared using the Mann-Whitney $U$ test. Categorical data are summarized by observed frequencies and percentages and compared using Fisher's exact test. Treatment effects were compared between both groups using differences in medians for continuous variables and odd ratios for categorical data (both with 95\% confidence intervals).
In case of multiple comparisons, a Bonferroni correction was performed. For the time to reach an Aldrete score 9, the Kaplan-Meier method was used.

In order to check for intellectual and psychosocial differences at baseline that could influence the neurocognitive outcome variables, a one-way ANOVA was performed.

For the neurocognitive tests, repeated measure analyses with group (control, xenon, and sevoflurane) as between- and time (baseline, 2 and 24 hours postoperatively) as within-variable were performed. Intelligence was entered as covariate because the control group scored significantly higher for IQ than both anesthesia groups. For significant interaction effects post hoc tests (Fisher's least significant differences) were performed. As a next step, logistic regression analyses were planned for the significant interaction effects to test whether these significant effects are clinical relevant. IQ and the use of anesthesia were used as predictors for clinical deviant performance ( $\geq 2 S D$ than age reference norms). Statistical significance was reached when the exact $P$-values were $<0.05$.

\section{3 | RESULTS}

\section{1 | Study flow, baseline characteristics, procedural characteristics, and risk category}

From November 2014 to November 2016, 40 children aged between 4 and 12 years old were included in this trial and randomized to receive either xenon-augmented sevoflurane (xenon group) or mono-sevoflurane (sevoflurane group) for the maintenance of the anesthesia (Figure 1). No significant differences were observed for demographic data (Table 1) or baseline clinical parameters except for $\mathrm{SpO}_{2}$, which was significantly lower in the xenon group (Table S1). There was no difference between both groups for the prevalence of PONV risk factors as published by Eberhart (including the following items: age $>3$ years, surgery $>30$ minutes, strabism surgery, history of PONV, or PONV in relatives). ${ }^{18}$

All patients underwent baseline testing and received the allocated intervention. Groups did not differ regarding the type of procedure (diagnostic or interventional), the procedural risk category (as defined by the "Congenital Cardiac Catheterization Project on Outcomes"19) or the duration of the procedure (Table 2). Twenty-two age- and gender-matched healthy and nonhospitalized children underwent the same neurocognitive tests at comparable time intervals.

\section{2 | Primary outcome}

\subsection{1 | Safety}

Between groups, intraprocedural HR and MAP were comparable. MAP in both groups differed significantly from BL throughout the procedure (Figure S1). The number of children that developed a change in HR or MAP of $20 \%$ (Table 2) and $30 \%$ (data not shown) at least once during the procedure did not differ statistically. Likewise, 


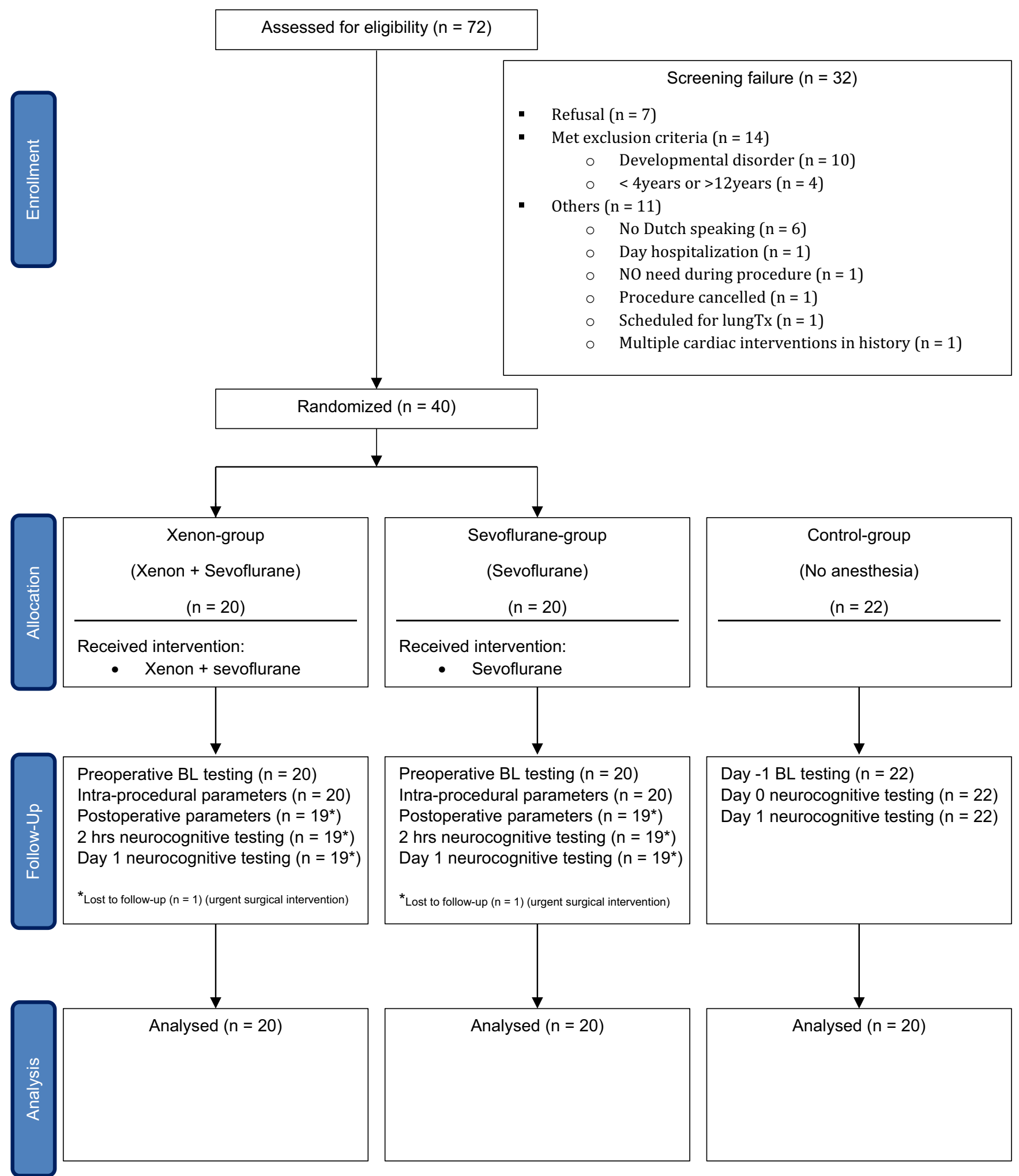

FIGURE 1 Flow diagram according to the CONSORT statement [Colour figure can be viewed at wileyonlinelibrary.com]

the time-weighted hypotensive episodes were comparable between both groups (Table 2). While there was no significant difference for the administration of phenylephrine, atropine, or fluids, ephedrine was required significantly less frequently and in significantly lower doses in the xenon group (Table 2).

\subsection{2 | Feasibility}

Median [IQR] BIS values at each time-point during the procedure and the overall mean BIS values ( $P$-value: $<0.0001)$ were higher in the xenon group (48 [42-57]) compared to the sevoflurane group (44 
TABLE 1 Demographic data

\begin{tabular}{|c|c|c|c|c|}
\hline & & Xenon + Sevoflurane & Sevoflurane & Control group \\
\hline \multicolumn{5}{|l|}{ Demographic data } \\
\hline Age & y & $7.5[4-10]$ & $7.5[5-9]$ & $8.0[5-10]$ \\
\hline Weight & $\mathrm{kg}$ & $21.6[18.4-31.1]$ & $22.5[18.6-25]$ & NA \\
\hline Height & $\mathrm{cm}$ & $122.7[110.2-143.8]$ & $127.5[113.2-133.5]$ & NA \\
\hline Gender (Female/male) & n (\%) & $8 / 12(40 / 60)$ & $9 / 11(45 / 55)$ & $11 / 11(50 / 50)$ \\
\hline \multicolumn{5}{|c|}{ Cognitive and psychosocial functioning } \\
\hline Intelligence & IQ-score & $108[102-115]$ & $92[78-106]$ & $106[90-122]$ \\
\hline Executive functioning (BRIEF) & T-value & $47[40-54]$ & $50[42-58]$ & $47[40-55]$ \\
\hline Internalizing problems (CBCL) & T-value & $56[48-65]$ & $52[46-59]$ & $48[36-60]$ \\
\hline Externalizing problems (CBCL) & T-value & $47[38-57]$ & $48[40-57]$ & $44[37-51]$ \\
\hline Total problem scale (CBCL) & T-value & 51 [41-61] & 49 [42-57] & 45 [39-51] \\
\hline
\end{tabular}

BRIEF, Behavioral Rating Inventory of Executive Functioning; CBCL, Child Behavior Checklist.

[36-49]) but remained within the limits of adequate depth of anesthesia (40-60) (Figure S2). No patients developed clinical signs of inappropriate depth of anesthesia.

The xenon group required significantly lower sevoflurane concentrations than the sevoflurane group to attain a comparable depth of anesthesia (Table 2). Mean inspiratory xenon concentrations were within the target range (50-65\%). Intraoperative opioid use was comparable in both groups (Table 2). Intraoperative respiratory parameters (endtidal $\mathrm{CO}_{2}$ and $\mathrm{SpO}_{2}$ ) remained within normal limits according to the child's pathology (data not shown).

\section{3 | Secondary outcomes}

\subsubsection{Neurocognitive outcome}

The control group differed significantly from both anesthesia groups for intelligence $(P=0.014)$. No significant group differences for psychosocial and executive functions were found (Table 1). Significant interaction effects were found for alertness (speed and variability), 2 hours after recovery from anesthesia: the two anesthesia groups performed worse for both measures of alertness than control subjects who had not been exposed to anesthesia: RT and variability of RT. For working memory, inhibition, cognitive flexibility, and all four motor coordination tasks, no significant interaction effects of anesthesia were found (Table 3). In order to investigate the clinical relevance of these significant effects, we used logistic regression analyses to predict clinical deviant performance ( $\geq 2 \mathrm{SD}$ than age reference norms). IQ and the use of anesthesia were used as predictors. The use of anesthesia was not a significant predictor for clinical deviant performance at baseline and 24 hours postoperatively. However, 2 hours postoperatively a borderline significant effect of anesthesia on alertness speed ( $P=0.065$, odds ratio 4.1$)$ and a significant effect on alertness variability ( $P=0.02$, odds ratio 5.8 ) were found. This effect was indifferent for both anesthesia regimens. Our data suggest a selective, temporarily, and clinically relevant impairment of alertness 2 hours after the intervention.

\subsection{2 | Recovery parameters}

Enhanced recovery was observed in the xenon group as indicated by a higher recovery index and shorter times to open eyes and to extubation (Table 4). Readiness for discharge and length of PACU stay were not statistically different for sevoflurane and xenon-augmented sevoflurane (Table 4).

Both treatment groups did not differ with respect to the incidence of ED. Neither the use of antiemetics nor the incidence of PONV was statistically different between both groups (Table 4).

\subsection{3 $\mid$ (S)AE}

Intraprocedural loss of intravascular device necessitating urgent surgery occurred during the procedure in two patients (one per group) and postoperatively in one patient (sevoflurane group). Likewise, the incidence of all other AEs was not statistically different between the groups.

\subsubsection{Laboratory results}

When compared to baseline, both groups showed a similar and statistically significant elevation of S100 $\beta$ and IL-10 at the end of the procedure (Figure 2). Blood levels of IL-6 remained unchanged (Figure 2).

\section{DISCUSSION}

The present trial shows that the adjuvant administration of $50-65 \mathrm{vol}-$ $\%$ xenon to sevoflurane anesthesia is feasible in school-aged children suffering from CHD and undergoing heart catheterization. While the addition of xenon was unable to reduce the incidence of periprocedural hemodynamic instability, there was a significant reduction in the use of ephedrine and a faster recovery. sevoflurane anesthesia resulted in a decreased alertness early after recovery, an effect from 
TABLE 2 Intraoperative data

\begin{tabular}{|c|c|c|c|c|c|c|}
\hline & & Xenon + Sevoflurane & Sevoflurane & $\begin{array}{l}\text { Difference in median } \\
(95 \% \mathrm{Cl})\end{array}$ & OR $(95 \% \mathrm{Cl})$ & $P$-value \\
\hline \multicolumn{7}{|l|}{ Procedural characteristics } \\
\hline Risk categorya: 1/2/3/4 & $\mathrm{n}(\%)$ & $2 / 7 / 7 / 4(10 / 35 / 35 / 20)$ & 4/7/9/0 (20/35/45/0) & - & - & 0.210 \\
\hline Duration of procedure & $\min$ & $69.0[46.2-88.7]$ & $53.5[46.0-72.7]$ & $-11(-28$ to 5$)$ & - & 0.203 \\
\hline \multicolumn{7}{|l|}{ Intraprocedural medication } \\
\hline Propofol & $\mathrm{mg} / \mathrm{kg}$ & $2.99[2.76-3.12]$ & $3.00[2.87-3.04]$ & $0.00(-0.12$ to 0.21$)$ & - & 0.962 \\
\hline Total fentanyl & $\mu \mathrm{g} / \mathrm{kg}$ & $2.11[2.01-2.94]$ & $2.68[2.01-3.00]$ & $0.125(-0.14$ to 0.72$)$ & - & 0.429 \\
\hline Rocuronium & $\mathrm{mg} / \mathrm{kg}$ & $0.30[0.30-0.33]$ & $0.31[0.30-0.33]$ & 0.003 (-0.01 to 0.02$)$ & - & 0.682 \\
\hline Ephedrine & $\mathrm{mg} / \mathrm{kg}$ & $0.00[0.00-0.00]$ & $0.00[0.00-0.02]$ & $0.00(0-0)$ & - & 0.047 \\
\hline \multirow[t]{2}{*}{ Atropine } & $\mathrm{n}(\%)$ & $1(5)$ & $1(5)$ & - & $0.900(0.05-16.59)$ & 1.000 \\
\hline & $\mu \mathrm{g} / \mathrm{kg}$ & $0.00[0.00-0.00]$ & $0.00[0.00-0.00]$ & $0.00(0-0)$ & - & 1.000 \\
\hline Xenon consumption & L & $10.20[9.02-12.57]$ & - & - & - & - \\
\hline $\begin{array}{l}\text { Mean inspiratory xenon } \\
\text { concentration }\end{array}$ & $\%$ & 55.35 [50.45-58.07] & - & - & - & - \\
\hline Sevoflurane consumption & $g$ & $2.43[1.26-3.35]$ & $5.02[2.87-8.52]$ & $2.385(1.09-4.36)$ & - & 0.000 \\
\hline $\begin{array}{l}\text { Mean inspiratory sevoflurane } \\
\text { concentration }\end{array}$ & $\%$ & $0.96[0.75-1.20]$ & $2.04[1.80-2.22]$ & $1.067(0.90-1.24)$ & - & 0.000 \\
\hline $\begin{array}{l}\text { Mean expiratory sevoflurane } \\
\text { concentration }\end{array}$ & $\%$ & $0.85[0.69-1.07]$ & $1.88[1.69-1.99]$ & $0.987(0.82-1.16)$ & - & 0.000 \\
\hline \multicolumn{7}{|l|}{ Fluid management } \\
\hline \multicolumn{7}{|c|}{ The incidence of hemodynamic instability } \\
\hline HR: $>20 \%$ change from $B L$ & $\mathrm{n}(\%)$ & $16(80)$ & $16(80)$ & $1.000(0.21-4.71)$ & - & 1.000 \\
\hline MAP: $>20 \%$ change from $B L$ & $\mathrm{n}(\%)$ & $19(95)$ & $20(100)$ & - & - & 1.000 \\
\hline $\begin{array}{l}\text { Vasopressors/inotropes/ } \\
\text { chronotropes }\end{array}$ & $\mathrm{n}(\%)$ & $3(15)$ & $7(35)$ & $0.328(0.07-1.52)$ & - & 0.273 \\
\hline $\begin{array}{l}\text { Patients fulfilling one of the } \\
\text { criteria }\end{array}$ & n (\%) & $19(95)$ & $20(100)$ & - & - & 1.000 \\
\hline
\end{tabular}

$\mathrm{BL}$, baseline; HR, heart rate; MAP, mean arterial pressure.

Data are presented as median [interquartile range] and as absolute numbers [n, with the percentage (\%) of the whole].

Bold indicates statistical significance.

aRisk of intervention assessed according to the 'Congenital Cardiac Catheterization Project on Outcomes'. ${ }^{19}$

which the patients fully recovered 24 hours postoperatively and that could not be countered by the addition of xenon.

The results of this study largely confirm our previous findings in younger children that xenon-augmented sevoflurane anesthesia is feasible in the pediatric population. ${ }^{8}$ In line with our observations in younger children and data from the adult population, ${ }^{20}$ the addition of xenon allows to reduce the concentrations of anesthetics to which xenon is added in order to attain a comparable depth of anesthesia, suggesting an additive or even synergistic anesthetic effect. Acknowledging the ongoing controversies regarding the reliability of BIS-monitoring in children, ${ }^{21}$ we titrated sevoflurane concentrations not only according to the obtained BIS values but also evaluated clinical signs suggestive for an inadequate depth of anesthesia (like increases in HR or movements).

Nearly all children of this trial developed at least one episode of hemodynamic instability (as defined by a $>20 \%$ change in HR 


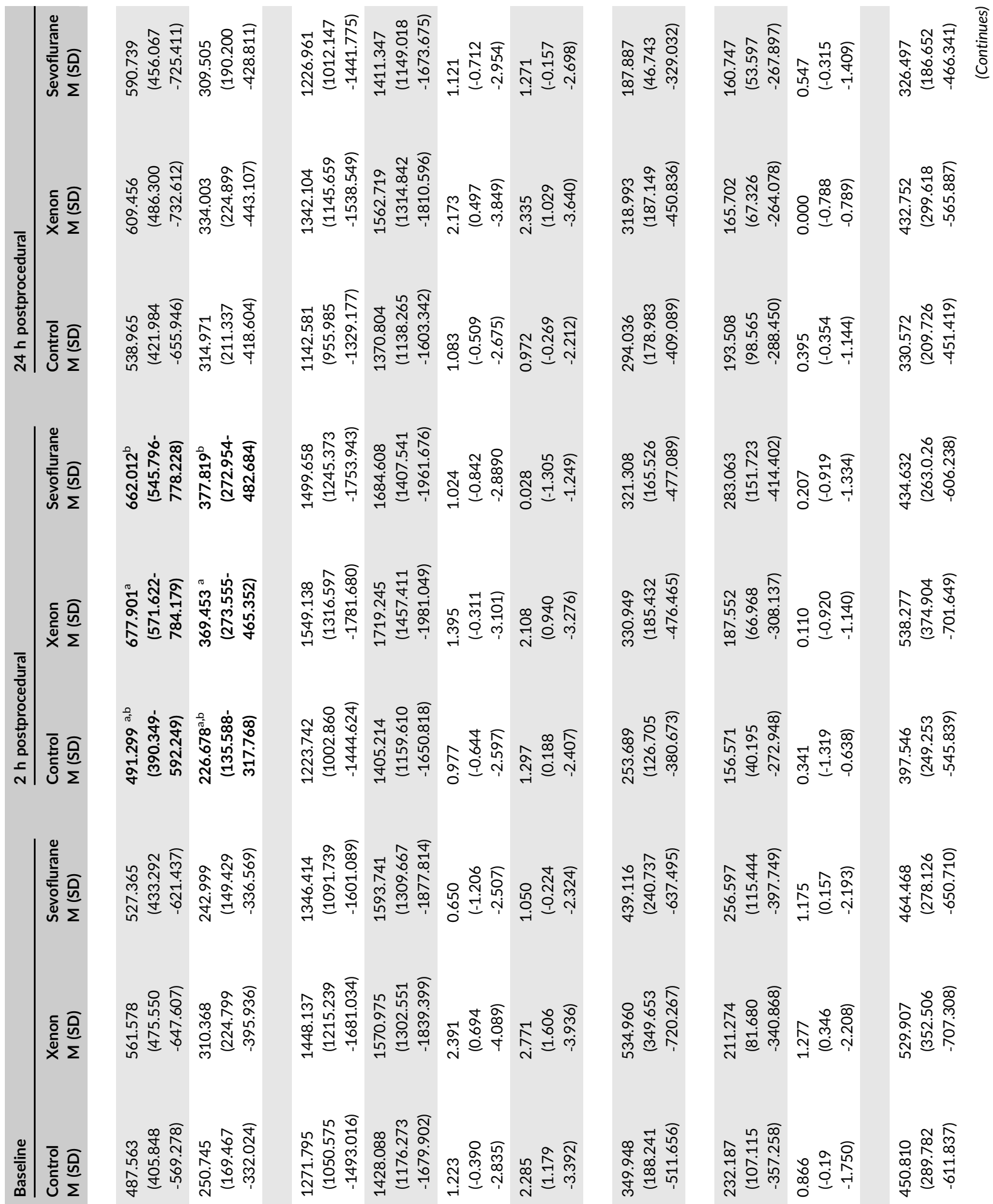

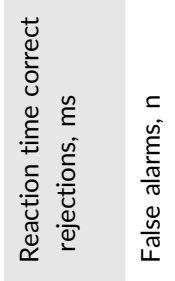



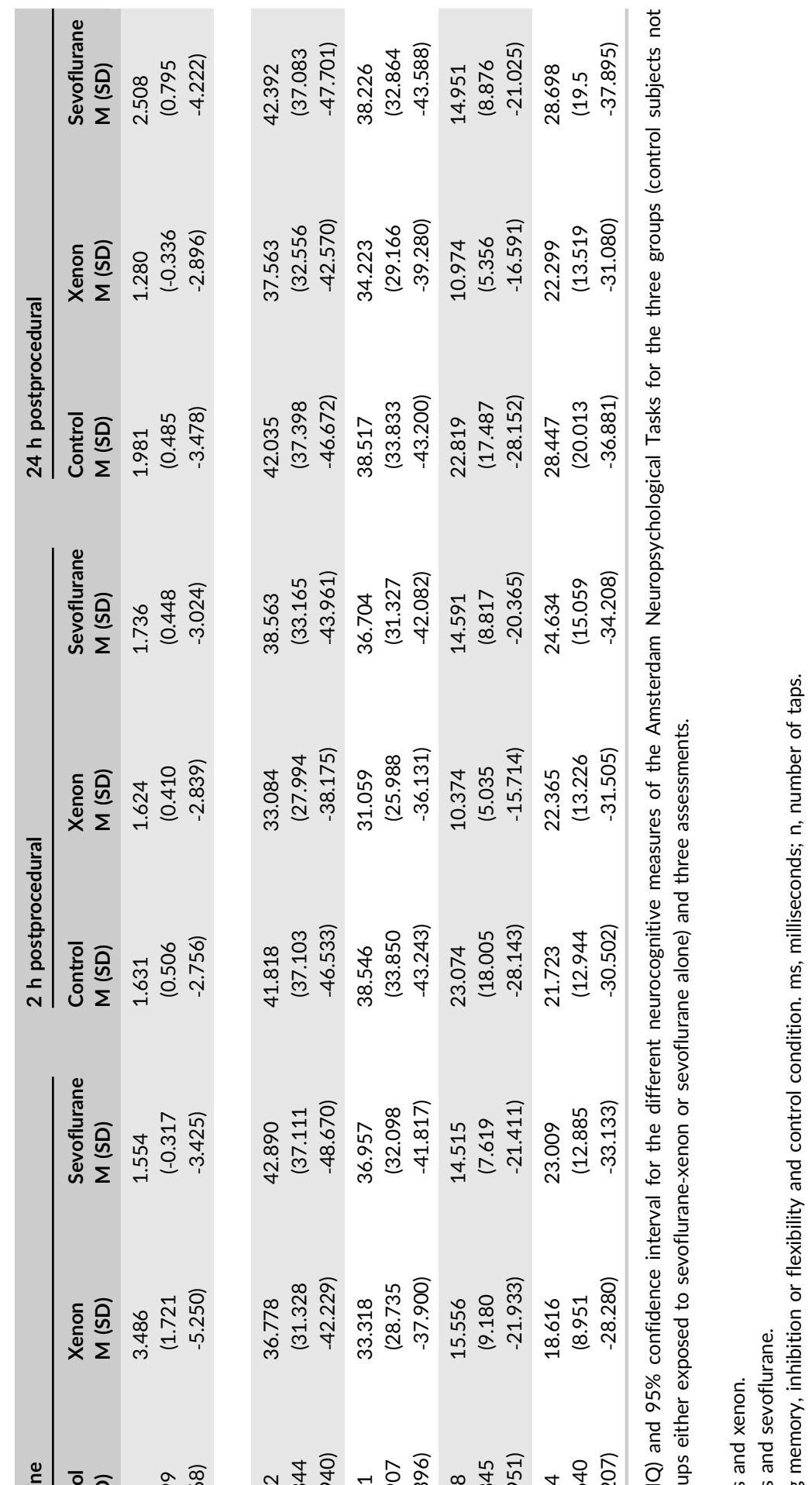

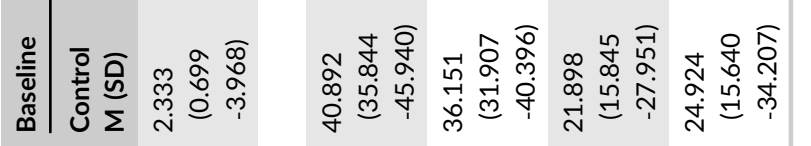

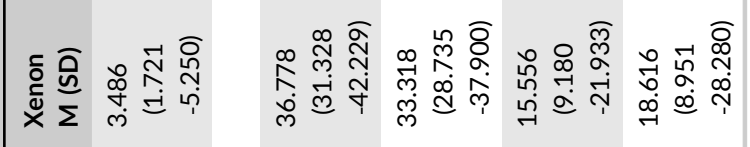

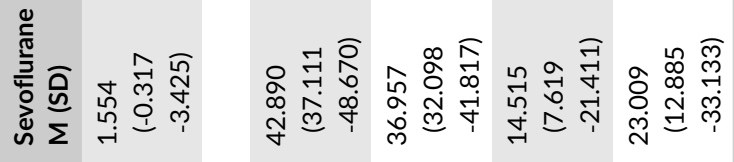

ơ

는 는

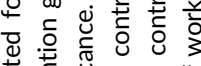


TABLE 4 Postprocedural data

\begin{tabular}{|c|c|c|c|c|c|}
\hline & & Xenon + sevoflurane & Sevoflurane & $\begin{array}{l}\text { Difference in median } \\
(95 \% \mathrm{Cl})\end{array}$ & $P$-value \\
\hline \multicolumn{6}{|l|}{ Recovery } \\
\hline Time to open eyes & $\min : s$ & 07:03 [04:51-09:00] & $14: 31$ [11:50-17:58] & $7.18(4.50-9.83)$ & 0.000 \\
\hline Recovery index & $/ \min$ & $0.52[0.41-0.78]$ & $0.25[0.20-0.33]$ & $-0.28(-0.41$ to -0.19$)$ & 0.000 \\
\hline T10 & & $1(5.3)$ & $3(15.8)$ & - & 0.604 \\
\hline T15 & & $0(0)$ & $3(15.8)$ & - & 0.230 \\
\hline T30 & & $2(10.5)$ & $2(10.5)$ & - & 1.000 \\
\hline $\mathrm{T} 45$ & & $1(5.3)$ & $2(10.5)$ & - & 1.000 \\
\hline Four-point agitation scale $\geq 3$ & & $2(10.5)$ & $3(15)$ & - & 1.000 \\
\hline PAEDS $\geq 12$ & & $0(0)$ & $2(10.5)$ & - & 0.486 \\
\hline PONV & n (\%) & $7(36.8)$ & $6(31.6)$ & - & 1.000 \\
\hline
\end{tabular}

Data are presented as median [IQR] or as absolute numbers [n, with the percentage (\%) of the whole].

PACU, postanesthesia care unit; PAEDS, pediatric anesthesia emergence delirium scale; PONV, postoperative nausea and vomiting.

Bold indicates statistical significance.

or MAP) and/or required a pharmacological hemodynamic intervention. This observation is in contrast with a variety of studies reporting an increased hemodynamic stability observed during xenon anesthesia in adults, ${ }^{1}$ but again in line with our findings in children $<4$ years old. ${ }^{8}$ The discrepancy between the adults and children with CHD may be due to several reasons. First, children with CHD undergoing cardiac catheterization are at increased risk for hemodynamic instability due to their underlying pathology and the intervention. ${ }^{22}$ Second, anesthesia with xenon alone is impossible to achieve in children. Notably, using a regression analysis of meta-analytic data, the MAC of xenon was fitted to be $92 \%$ in children at the age of 1 year. ${ }^{7}$ As a consequence, a maximum of approximately $2 / 3$ MAC of xenon can be administered when hypoxia is to be avoided. Therefore, relatively high concentrations of other anesthetics (in our trial: sevoflurane) are still needed to provide an adequate depth of anesthesia when using xenon in children. We suggest that the beneficial hemodynamic effects of xenon may have been masked by the concomitant use of sevoflurane in our population. Interestingly, the adjuvant use of xenon still resulted in reduced requirements for ephedrine, which may facilitate intraoperative management of this vulnerable patient population. This finding confirms previous reports from other settings. ${ }^{2,3,8}$ While we are unable to completely rule out an interaction between the depth of anesthesia and the occurrence of hypotension, our data suggest that sevoflurane anesthesia is intrinsically associated with an increased risk of hypotension as compared to the combination of sevoflurane with xenon (and this independently from the anesthetic plane). Interestingly, while sevoflurane patients required a total of nine ephedrine administrations to maintain predefined hemodynamic goals, only three of these administrations were necessary when the BIS was significantly lower than in the xenon group (at T10, T20, and T25, see Table S2). The other six ephedrine administrations were performed at time-points at which both groups did not differ with respect to the BIS. Please also note that the majority of ephedrine administrations had to be performed despite the BIS being $>40$, ie, in the range of an appropriate depth of anesthesia. In contrast, not a single xenon patient required ephedrine for the treatment of hypotension, even when the BIS was below 40 (as was the case in 10 out of 20 patients for at least one episode of 5 minutes).

In our study, addition of xenon resulted in an enhanced recovery as demonstrated by several parameters established to quantify speed and quality of recovery. This finding was expected from experience in adults ${ }^{1}$ and in younger children ${ }^{8}$ and can be explained by the low blood/gas partition coefficient of xenon. Rapid emergence (as seen after sevoflurane anesthesia) has been suggested to play an important role in the pathophysiology of ED. ${ }^{12}$ Despite the faster emergence, children in the xenon group had no increased incidence of ED. Remarkably, we could not confirm a statistically significantly increased incidence of PONV in the xenon group, which is in contrast to the adult population.

The results of the neurocognitive tests performed in this study replicate previous findings showing significant neurocognitive impairments early after sevoflurane anesthesia. Several studies showed that RT measures and tasks measuring alertness or psychomotor speed were temporarily affected after anesthesia, ${ }^{15-17}$ but recovered after 24 hours. While xenon has been demonstrated to exert neuroprotection in various models of neuronal injury, ${ }^{5,23,24}$ our findings do not support the hypothesis that xenon might protect from early postoperative sevoflurane-induced neurocognitive impairment. In our 

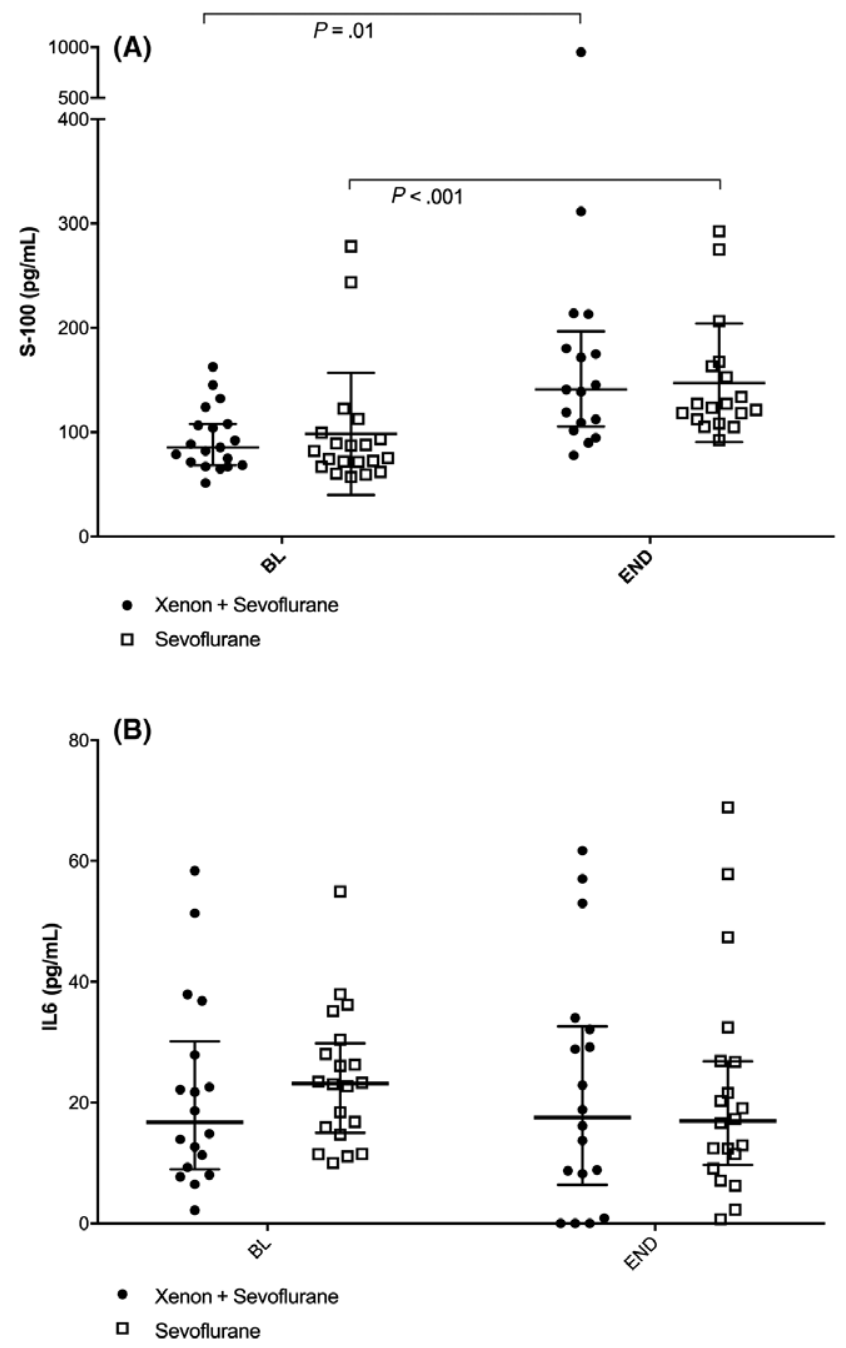

(C)

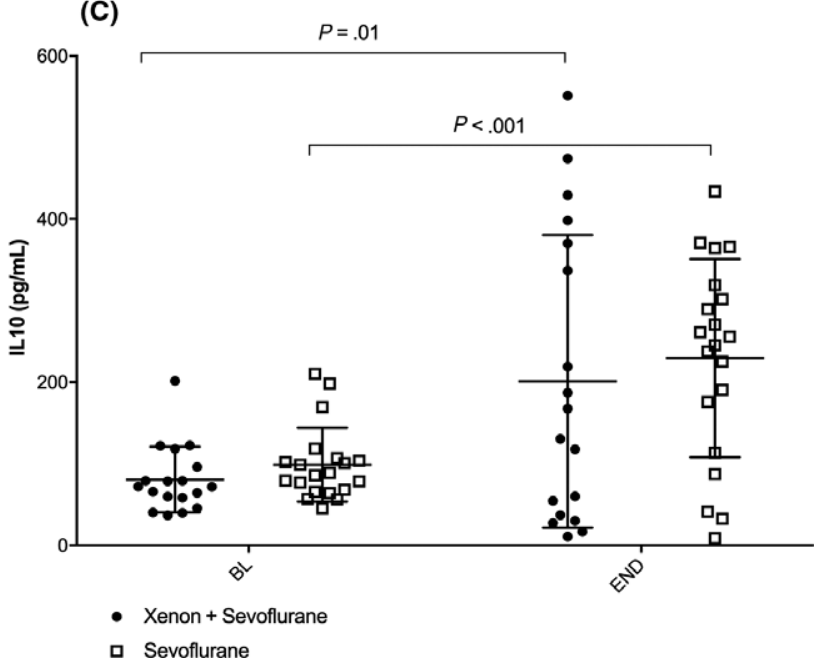

FIGURE 2 Intraoperative time course of (A) serum protein S100 $\beta$, (B) IL 6 and (C) IL 10

patients, alertness was affected 2 hours, but not 24 hours postoperatively irrespective of the treatment allocation, suggesting that both anesthesia regimens had a temporary and comparable effect on neurocognitive performance. Alertness is a crucial attentional network, necessary to achieve and maintain a state of sensitivity to incoming information. Therefore, an unimpaired alerting network is pivotal for efficient and quick responding to incoming information.

Interestingly, the observed neurocognitive impairment was paralleled by an increase in blood levels of $\mathrm{S} 100 \beta$. $\mathrm{S} 100 \beta$ is an astroglial receptor protein involved in the regulation of different vital intracellular processes such as neuronal differentiation, axonal growth, and calcium homeostasis. ${ }^{25}$ The release of $\mathrm{S} 100 \beta$ into peripheral blood has been linked to an increased permeability of the blood-brain barrier and was found to be significantly increased after neurotrauma, cerebral ischemia, cardiac arrest, and cardiac surgery. ${ }^{26}$ Moreover, in adult cardiac surgery, high $\mathrm{S} 100 \beta$-levels were found to be correlated with adverse neurological and neuropsychological outcomes. In neonates with CHD, S100 $\beta$-levels were demonstrated to be inversely related to cerebral blood flow and to mortality. ${ }^{27}$ The mechanisms underlying the $\mathrm{S} 100 \beta$-increase during surgery remain elusive but may be related to neuroinflammatory processes with astrocytes releasing $\mathrm{S} 100 \beta$ upon stimulation by cytokines originating from systemic inflammation. We also observed a significant increase in IL-10 levels in all our patients. While these observations are certainly intriguing, they merely describe associations and should not be misinterpreted as a proof of causation.

We acknowledge that our study is subject to several limitations.

First, the anesthesiologist responsible for the conduct of anesthesia could not be blinded for the interventional treatment. Hemodynamic treatment was at his discretion but had to be triggered by a decrease in HR or MAP $>20 \%$ from baseline. A more rigorous algorithm to standardize these hemodynamic interventions would have likely reduced the risk for treatment bias, but is nearly impossible to design in children with diverse forms of CHD, especially since there is not even a consensus of a 'normal blood pressure' in a 'normal' child. The efficacy of xenon anesthesia in children undergoing cardiac catheterization could be most explicitly tested by comparing the number of hypotensive episodes and the degree of hypotension in the control group. However, such a direct quantification of hypotensive episodes would be unethical since hypotension is associated with poor outcome and should be corrected promptly. Consequently, we assessed hemodynamic stability by the number of hemodynamic interventions and the average dose of the atropine/ephedrine/phenylephrine that had to be administered to maintain the predefined intraoperative hemodynamic goals. This approach has been previously described and uses vasopressor doses as a surrogate and quantitative marker for hypotension. ${ }^{2}$ The fact that children treated with sevoflurane alone received more ephedrine is consequently interpreted as an indicator of less hemodynamic stability. It was exactly that increase in ephedrine administration that resulted in a hemodynamic profile indistinguishable from the xenon group.

Second, it is not justified to extrapolate the feasibility of xenonanesthesia in school-aged children undergoing cardiac catheterization to surgical settings with severe stimulation.

Third, at the start of this trial, the evidence on the use of xenonanesthesia in the pediatric population was scarce and no data for a 
power calculation were available. Consequently, this study was planned as a pilot study for xenon anesthesia in school-aged children and the relatively small sample size could affect the power of all outcome parameters.

Fourth, our control group for the neurocognitive evaluation was healthy and not hospitalized. Although no differences in baseline cognitive tests between the three groups were observed, the lack of control for hospitalization or the perspective of a medical intervention may present a significant bias.

Fifth, the validity of the BIS monitor has never been formally tested in children undergoing combined anesthesia with xenon and sevoflurane. However, we would like to note that the available evidence suggests that the BIS is a suitable instrument for assessing depth of xenon anesthesia. Fahlenkamp et $\mathrm{al}^{28}$ showed that during balanced xenon anesthesia, BIS values correlated well with the clinical assessment of hypnotic depth and were within the range recommended for adequate anesthesia. Stoppe et $\mathrm{al}^{29}$ demonstrated that the BIS showed a similar response to sevoflurane as to xenon anesthesia. In this study, the depth of anesthesia was additionally confirmed by the measurement of auditory evoked potentials. Last, the profile of the EEG changes observed during xenon anesthesia was found to closely resemble those caused by propofol. ${ }^{30}$ Furthermore, although age-related changes in BIS values in children were observed, it has been suggested that the BIS can be reliably used to monitor depth of anesthesia in children older than 1 year of age, showing better performance with increasing age. ${ }^{31,32}$ We therefore consider the BIS (together with interpretations of clinical signs) a valuable monitor of depth of anesthesia in our patient population.

In conclusion, xenon-augmented sevoflurane anesthesia in this small sample of school-aged children was feasible and resulted in faster emergence than sevoflurane anesthesia. sevoflurane anesthesia was associated with decreased alertness 2 hours after recovery from anesthesia and an increase in serum S100 $\beta$ and IL-10 with the addition of xenon however being unable to prevent this effect.

\section{ETHICAL APPROVAL}

The study protocol was approved by the local ethics committee (SR56902, Commissie Medische Ethiek, Universitaire Ziekenhuizen Leuven, September 17th, 2014) and by the Federal Agency for Medicines and Health Products, Brussels, Belgium (AFMPS/R\&D/ CED/mm830076, September 5th, 2014). The study is registered at the European Medicines Agency (EudraCT: 2014-002510-23) and reported according to the CONSORT statement.

\section{CONFLICT OF INTEREST}

S.R. has been supported by an unrestricted research grant from AirLiquide Belgium, a research grant of AirLiquide France and the Foundation Annie Planckaert-Dewaele (Biomedical Sciences Group, KU Leuven). Roche Diagnostics Belgium offered the S-100 kit. None of the funding institutions had influence on the design, analysis, or publication of the study.

\section{ORCID}

Sarah Devroe iD http://orcid.org/0000-0003-0931-7891

\section{REFERENCES}

1. Law LS-C, Lo EA-G, Gan TJ. Xenon anesthesia: a systematic review and meta-analysis of randomized controlled trials. Anesth Analg 2016;122:678-697.

2. Al Tmimi L, Van Hemelrijck J, van de Velde M, Sergeant P, Meyns B, Missant $\mathrm{C}$, et al. Xenon anaesthesia for patients undergoing offpump coronary artery bypass graft surgery: a prospective randomized controlled pilot trial. Br J Anaesth. 2015;115:550-559.

3. Al Tmimi L, Devroe S, Dewinter G, van de Velde M, Poortmans G, Meyns B, et al. Xenon as an adjuvant to propofol anesthesia in patients undergoing off-pump coronary artery bypass graft surgery: a pragmatic randomized controlled clinical trial. Anesth Analg. 2017;125:1118-1128.

4. De Deken J, Rex S, Monbaliu D, Pirenne J, Jochmans I. The efficacy of noble gases in the attenuation of ischemia reperfusion injury: a systematic review and meta-analyses. Crit Care Med. 2016;44:e886-e896.

5. Maze M. Preclinical neuroprotective actions of xenon and possible implications for human therapeutics: a narrative review. Can J Anaesth. 2016;63:212-226.

6. Alam A, Suen KC, Hana Z, Sanders RD, Maze M, Ma D. Neuroprotection and neurotoxicity in the developing brain: an update on the effects of dexmedetomidine and xenon. Neurotoxicol Teratol. 2017;60:102-116

7. Mapleson WW. Effect of age on MAC in humans: a meta-analysis. $\mathrm{Br}$ J Anaesth. 1996;76:179-185.

8. Devroe S, Meeusen R, Gewillig M, Cools B, Poesen K, Sanders R, et al. Xenon as an adjuvant to sevoflurane anesthesia in children younger than 4 years of age, undergoing interventional or diagnostic cardiac catheterization: A randomized controlled clinical trial. Pediatr Anesth. 2017:27:1210-1219.

9. Devroe S, Lemiere J, van de Velde M, Gewillig M, Boshoff D, Rex S. Safety and feasibility of xenon as an adjuvant to sevoflurane anaesthesia in children undergoing interventional or diagnostic cardiac catheterization: study protocol for a randomised controlled trial. Trials. $2015 ; 16: 74$.

10. de Sonneville L. Amsterdam Neuropsychological Tasks: Manual (Handleiding). Amsterdam, The Netherlands: Boom Test Uitgevers; 2011.

11. Rossaint R, Reyle-Hahn M, Schulte Am Esch J, Scholz J, Scherpereel $\mathrm{P}$, Vallet $\mathrm{B}$, et al. Multicenter randomized comparison of the efficacy and safety of xenon and isoflurane in patients undergoing elective surgery. Anesthesiology. 2003;98:6-13.

12. Bajwa SA, Costi D, Cyna AM. A comparison of emergence delirium scales following general anesthesia in children. Pediatr Anesth. 2010;20:704-711.

13. Al Tmimi L, van de Velde M, Herijgers P, Meyns B, Meyfroidt G, Milisen $\mathrm{K}$, et al. Xenon for the prevention of postoperative delirium in cardiac surgery: study protocol for a randomized controlled clinical trial. Trials. 2015;16:449.

14. Stoppe C, Fahlenkamp AV, Rex S, Veeck NC, Gozdowsky SC, Schälte $\mathrm{G}$, et al. Feasibility and safety of xenon compared with sevoflurane anaesthesia in coronary surgical patients: a randomized controlled pilot study. Br J Anaesth. 2013;111:406-416.

15. Chung F, Kayumov L, Sinclair DR, Edward R, Moller HJ, Shapiro CM. What is the driving performance of ambulatory surgical patients after general anesthesia? Anesthesiology. 2005;103:951-956.

16. Millar K, Asbury AJ, Bowman AW, Hosey MT, Musiello T, Welbury $R R$. The effects of brief sevoflurane-nitrous oxide anaesthesia upon children's postoperative cognition and behaviour. Anaesthesia. 2006;61:541-547. 
17. Millar K, Bowman AW, Burns D, McLaughlin P, Moores T, Morton NS, et al. Children's cognitive recovery after day-case general anesthesia: a randomized trial of propofol or isoflurane for dental procedures. Pediatr Anesth. 2014;24:201-207.

18. Eberhart LHJ, Geldner G, Kranke P, Morin AM, Schäuffelen A, Treiber $\mathrm{H}$, et al. The development and validation of a risk score to predict the probability of postoperative vomiting in pediatric patients. Anesth Analg. 2004;99:1630-1637.

19. Bergersen L, Gauvreau K, Marshall A, Kreutzer J, Beekman R, Hirsch $\mathrm{R}$, et al. Procedure-type risk categories for pediatric and congenital cardiac catheterization. Circ Cardiovasc Interv. 2011;4:188-194.

20. Nakata $Y$, Goto T, Ishiguro $Y$, Terui K, Kawakami H, Santo M, et al. Minimum alveolar concentration (MAC) of xenon with sevoflurane in humans. Anesthesiology. 2001;94:611-614.

21. Sadhasivam S, Ganesh A, Robison A, Kaye R, Watcha MF. Validation of the bispectral index monitor for measuring the depth of sedation in children. Anesth Analg. 2006;102:383-388.

22. Jayaram N, Beekman RH, Benson L, Holzer R, Jenkins K, Kennedy $\mathrm{KF}$, et al. Adjusting for risk associated with pediatric and congenital cardiac catheterization: a report from the NCDR IMPACT registry. Circulation. 2015;132:1863-1870.

23. Chakkarapani E, Dingley J, Liu X, Hoque N, Aquilina K, Porter H, et al. Xenon enhances hypothermic neuroprotection in asphyxiated newborn pigs. Ann Neurol. 2010;68:330-341.

24. Zhuang L, Yang T, Zhao H, Fidalgo AR, Vizcaychipi MP, Sanders RD, et al. The protective profile of argon, helium, and xenon in a model of neonatal asphyxia in rats. Crit Care Med. 2012;40:1724-1730.

25. Al Tmimi L, van de Velde M, Meyns B, Meuris B, Sergeant P, Milisen $\mathrm{K}$, et al. Serum protein S100 as marker of postoperative delirium after off-pump coronary artery bypass surgery: secondary analysis of two prospective randomized controlled trials. Clin Chem Lab Med. 2016;54:1671-1680.

26. Abu-Sultaneh S, Hehir DA, Murkowski K, Ghanayem NS, Liedel J, Hoffmann RG, et al. Changes in cerebral oxygen saturation correlate with $\mathrm{S} 100 \mathrm{~B}$ in infants undergoing cardiac surgery with cardiopulmonary bypass. Pediatr Crit Care Med. 2014;15:219-228.

27. Bokesch PM, Appachi E, Cavaglia M, Mossad E, Mee RBB. A glialderived protein, $\mathrm{S} 100 \mathrm{~B}$, in neonates and infants with congenital heart disease: evidence for preexisting neurologic injury. Anesth Analg. 2002;95:889-892.
28. Fahlenkamp AV, Peters D, Biener IA, Billoet C, Apfel CC, Rossaint R, et al. Evaluation of bispectral index and auditory evoked potentials for hypnotic depth monitoring during balanced xenon anaesthesia compared with sevoflurane. Br J Anaesth. 2010;105:334-341.

29. Stoppe C, Peters D, Fahlenkamp AV, Cremer J, Rex S, Schälte G, et al. aepEX monitor for the measurement of hypnotic depth in patients undergoing balanced xenon anaesthesia. $\mathrm{Br} J$ Anaesth. 2012;108:80-88.

30. Laitio RM, Kaskinoro K, Särkelä MOK, Kaisti KK, Salmi E, Maksimow $\mathrm{A}$, et al. Bispectral index, entropy, and quantitative electroencephalogram during single-agent xenon anesthesia. Anesthesiology. 2008;108:63-70.

31. Davidson AJ, Kwok T. Performance of BIS in children using the paediatric BIS Quattro sensor. Anaesth Intensive Care. 2008;36:807-813.

32. Sciusco A, Standing JF, Sheng Y, Raimondo P, Cinnella G, Dambrosio $M$. Effect of age on the performance of bispectral and entropy indices during sevoflurane pediatric anesthesia: a pharmacometric study. Pediatr Anesth. 2017;27:399-408.

\section{SUPPORTING INFORMATION}

Additional supporting information may be found online in the Supporting Information section at the end of the article.

How to cite this article: Devroe S, Lemiere J, Van Hese L, et al. The effect of xenon-augmented sevoflurane anesthesia on intraoperative hemodynamics and early postoperative neurocognitive function in children undergoing cardiac catheterization: A randomized controlled pilot trial. Pediatr Anesth. 2018;28:726-738. https://doi.org/

10.1111/pan.13444 